PROCEEDINGS OF THE

AMERICAN MATHEMATICAL SOCIETY

Volume 130, Number 6, Pages 1681-1688

S 0002-9939(01)06237-2

Article electronically published on October 24, 2001

\title{
REMOVABLE SETS FOR CONTINUOUS SOLUTIONS OF QUASILINEAR ELLIPTIC EQUATIONS
}

\author{
TERO KILPELÄINEN AND XIAO ZHONG \\ (Communicated by Juha M. Heinonen)
}

\begin{abstract}
We show that sets of $n-p+\alpha(p-1)$ Hausdorff measure zero are removable for $\alpha$-Hölder continuous solutions to quasilinear elliptic equations similar to the $p$-Laplacian. The result is optimal. We also treat larger sets in terms of a growth condition. In particular, our results apply to quasiregular mappings.
\end{abstract}

\section{INTRODUCTION}

Throughout this paper we let $\Omega$ be an open set in $\mathbf{R}^{n}$ and $1<p<\infty$ a fixed number. Continuous solutions $u \in W_{\mathrm{loc}}^{1, p}(\Omega)$ of the equation

$$
-\operatorname{div} \mathcal{A}(x, \nabla u)=0
$$

are called $\mathcal{A}$-harmonic in $\Omega$. Here $\mathcal{A}: \mathbf{R}^{n} \times \mathbf{R}^{n} \rightarrow \mathbf{R}^{n}$ is assumed to verify for some constants $0<\lambda \leq \Lambda<\infty$ :

the function $x \mapsto \mathcal{A}(x, \xi)$ is measurable for all $\xi \in \mathbf{R}^{n}$, and

the function $\xi \mapsto \mathcal{A}(x, \xi)$ is continuous for a.e. $x \in \mathbf{R}^{n}$;

for all $\xi \in \mathbf{R}^{n}$ and a.e. $x \in \mathbf{R}^{n}$

$$
\begin{gathered}
\mathcal{A}(x, \xi) \cdot \xi \geq \lambda|\xi|^{p}, \\
|\mathcal{A}(x, \xi)| \leq \Lambda|\xi|^{p-1}, \\
(\mathcal{A}(x, \xi)-\mathcal{A}(x, \zeta)) \cdot(\xi-\zeta)>0,
\end{gathered}
$$

whenever $\xi \neq \zeta$. A prime example of the operators is the $p$-Laplacian

$$
-\Delta_{p} u=-\operatorname{div}\left(|\nabla u|^{p-2} \nabla u\right) .
$$

In this case, the continuous solutions of (1.1) are called $p$-harmonic functions. The main result in this paper is the following theorem.

Received by the editors September 13, 2000 and, in revised form, December 1, 2000

2000 Mathematics Subject Classification. Primary 35J60, 35J70, 30C65.

Key words and phrases. $p$-Laplacian, equations involving measures, removable sets, quasiregular mappings.

This research was supported by the Academy of Finland (Project \#41964).

(C)2001 American Mathematical Society 
1.6. Theorem. Let $E \subset \Omega$ be closed and $s>0$. Suppose that $u$ is a continuous function in $\Omega, \mathcal{A}$-harmonic in $\Omega \backslash E$ such that

$$
\left|u\left(x_{0}\right)-u(y)\right| \leq C\left|x_{0}-y\right|^{(s+p-n) /(p-1)}
$$

for all $y \in \Omega$ and $x_{0} \in E$. If $E$ is of $s$-Hausdorff measure zero, then $u$ is $\mathcal{A}$-harmonic in $\Omega$.

Since sets of $p$-capacity zero are removable for bounded $\mathcal{A}$-harmonic functions, Theorem 1.6 is interesting for $s>n-p$ only. Kilpeläinen, Koskela, and Martio [KKM had a special version of Theorem 1.6, where $u$ was assumed to be flat on $E$ and Hausdorff measure was replaced by a Minkowski content type condition.

1.8. Corollary. Suppose that $u \in C^{0, \alpha}(\Omega), 0<\alpha \leq 1$, is $\mathcal{A}$-harmonic in $\Omega \backslash E$. If $E$ is a closed set of $n-p+\alpha(p-1)$ Hausdorff measur 1 zero, then $u$ is $\mathcal{A}$-harmonic in $\Omega$.

The following theorem shows that Corollary 1.8 is optimal. Before stating the theorem, we recall that there is a constant $\kappa, 0<\kappa=\kappa(n, p, \lambda, \Lambda) \leq 1$, such that every $\mathcal{A}$-harmonic function $h$ in $\Omega$ verifies the local Hölder continuity estimate

$$
\operatorname{osc}(h, B(x, r)) \leq c\left(\frac{r}{R}\right)^{\kappa} \operatorname{osc}(h, B(x, R))
$$

for each $0<r<R$ and $B(x, R) \subset \Omega$ [HKM, 6.6]. For smooth $\mathcal{A}$, in particular for the $p$-Laplacian, we may choose $\kappa=1$ (see e.g. [K, 2.3]).

1.10. Theorem. Let $\kappa$ be as above and $0<\alpha<\kappa$. Suppose that $E \subset \Omega$ is a closed set with positive $n-p+\alpha(p-1)$ Hausdorff measure. ${ }^{1}$ Then there is $u \in C^{0, \alpha}(\Omega)$ which is $\mathcal{A}$-harmonic in $\Omega \backslash E$, but does not have an $\mathcal{A}$-harmonic extension to $\Omega$.

For the $p$-Laplacian we have the following sharp result.

1.11. Corollary. Let $0<\alpha<1$. A closed set $E$ is removable for $\alpha$-Hölder continuous $p$-harmonic functions if and only if $E$ is of $n-p+\alpha(p-1)$ Hausdorff measure ${ }^{1}$ zero.

Carleson [C] proved Corollary 1.11 for the Laplacian $(p=2)$. As to the quasilinear case, Heinonen and Kilpeläinen [HK, 4.5] proved Corollary 1.8 with $\alpha=1$, and Trudinger and Wang [TW] proved it under the assumption that $u$ has an $\mathcal{A}$ superharmonic extension to $\Omega$, which can be dispensed with for small $\alpha$. However, in the general situation the growth condition of Theorem 1.6 yields a more useful result, since $\mathcal{A}$-harmonic functions are not in general in $C^{0, \alpha}$ for $\alpha$ close to 1 . Koskela and Martio KM2 proved a weaker version of Corollary 1.13 and 1.8, where Minkowski content is used in place of Hausdorff measure. Buckley and Koskela BK] also established very special cases of Corollary 1.8. In $[\mathrm{K}]$ there is a weaker version of Theorem 1.10 .

A mapping $f: \Omega \rightarrow \mathbf{R}^{n}$ is called quasiregular if $f \in W_{\text {loc }}^{1, n}(\Omega)$ and there is a constant $K$ such that

$$
\left|f^{\prime}(x)\right|^{n} \leq K J_{f}(x)
$$

for a.e. $x \in \Omega$; here $J_{f}(x)$ is the Jacobian determinant of $f$ at $x$. The coordinate functions of a quasiregular map $f$ satisfy an equation of type (1.1) with $p=n$ (cf. [HKM, Ch. 14]), whence we have:

\footnotetext{
${ }^{1}$ Assume, of course, that $\alpha \geq(p-n) /(p-1)$.
} 
1.12. Corollary. Let $E \subset \Omega$ be a closed set of $s$-Hausdorff measure zero, $0<s \leq$ $n$. Suppose that $f: \Omega \rightarrow \mathbf{R}^{n}$ is a continuous mapping, quasiregular in $\Omega \backslash E$. If

$$
\left|f\left(x_{0}\right)-f(y)\right| \leq C\left|x_{0}-y\right|^{s /(n-1)}
$$

for all $y \in \Omega$ and $x_{0} \in E$, then $f$ is quasiregular in $\Omega$.

1.13. Corollary. Suppose that $f \in C^{0, \alpha}(\Omega)$ is quasiregular in $\Omega \backslash E$. If $E$ is a closed set of $\alpha(n-1)$-Hausdorff measure zero, then $f$ is quasiregular in $\Omega$.

Koskela and Martio KM1 showed that sets whose Minkowski dimension is less than $\alpha n$ are removable for $\alpha$-Hölder continuous quasiregular mappings provided that $\alpha<1-1 / n$, and the same for sets of $\alpha n$-Hausdorff measure zero if $\alpha \leq 1 / n$.

Our method of proof combines some ideas from $[\mathrm{K}], \mathrm{L}]$, and $[\mathrm{TW}$. We use solutions of equations

$$
-\operatorname{div} \mathcal{A}(x, \nabla u)=\mu,
$$

where $\mu$ is a nonnegative Radon measure from $W_{\text {loc }}^{-1, p^{\prime}}(\Omega)$, i.e. $u \in W_{\mathrm{loc}}^{1, p}(\Omega)$ and

$$
\int_{\Omega} \mathcal{A}(x, \nabla u) \cdot \nabla \varphi d x=\int_{\Omega} \varphi d \mu
$$

for all $\varphi \in C_{0}^{\infty}(\Omega)$. In particular, we prove the following theorem that improves the main theorem in $[\mathrm{K}]$.

1.14. Theorem. Let $\kappa$ be the number given by (1.9). Suppose that $u \in W_{\mathrm{loc}}^{1, p}(\Omega)$ is a solution of

$$
-\operatorname{div} \mathcal{A}(x, \nabla u)=\mu,
$$

where $\mu$ is a nonnegative Radon measure such that there are constants $M>0$ and $0<\alpha<\kappa$ with

$$
\mu(B(x, r)) \leq M r^{n-p+\alpha(p-1)}
$$

whenever $B(x, 3 r) \subset \Omega$. Then $u \in C^{0, \alpha}(\Omega)$. Moreover, $\kappa(n, p, 1,1)=1$, that is, in the case of the $p$-Laplacian any $\alpha<1$ will do.

Theorem 1.14 is the best possible (see [KM, 4.18], [K, 2.7]).

Finally, we remark here that Corollary 1.11 is not true when $\alpha=1$. The problem for which sets are removable for Lipschitz continuous $p$-harmonic functions is more delicate. David and Mattila [DM] treated the case $n=p=2$ : a compact set $E$ of finite 1-Hausdorff measure is removable for Lipschitz continuous harmonic functions if and only if $E$ is purely unrectifiable. The other cases remain open.

\section{Proof of Theorem 1.6}

We need a potential theoretic version of the obstacle problem. Suppose that $\psi$ is a continuous function on $\Omega$ and let the balayage $\hat{R}^{\psi}=\hat{R}^{\psi}(\Omega)$ be the pointwise infimum of all supersolutions 23 to (1.1) that lie above $\psi$ in $\Omega$. Similarly, let $\underline{\hat{R}}^{\psi}=\underline{\hat{R}}^{\psi}(\Omega)$ be the pointwise supremum of all subsolutions that lie below $\psi$ in $\Omega$.

\footnotetext{
${ }^{2}$ I.e. $u \in W_{\text {loc }}^{1, p}(\Omega)$ and $-\operatorname{div} \mathcal{A}(x, \nabla u) \geq 0$ in $\Omega$.
} 
Then $\hat{R}^{\psi} \geq \psi$ is a continuous supersolution in $\Omega$ and $\mathcal{A}$-harmonic in $\left\{\hat{R}^{\psi}>\psi\right\}$; similar statements hold for $\underline{\hat{R}}^{\psi}$. For a more thorough discussion see [HKM, Ch. 9]. Next we show the following estimate for the balayage; see $[\mathrm{L}]$ for a related result.

2.1. Lemma. Let $K \subset \Omega$ be compact. Suppose that $\psi$ is a continuous function with

$$
|\psi(x)-\psi(y)| \leq M|x-y|^{\alpha} \text { for all } x \in K \text { and } y \in \Omega,
$$

where $M>0$ and $\alpha>0$. Let $u=\hat{R}^{\psi}$ and

$$
\mu=-\operatorname{div} \mathcal{A}(x, \nabla u) .
$$

Then

$$
\mu(B(x, r)) \leq c r^{n-p+\alpha(p-1)}
$$

for all $r<r_{0}=\frac{1}{64} \operatorname{dist}(K, \partial \Omega)$ and $x \in K$; here $c=c(n, p, \lambda, \Lambda, M, \alpha)>0$.

Proof. Write

$$
I=\{x \in \Omega: \psi(x)=u(x)\}
$$

for the contact set.

First, let $x_{0} \in I$. We assume, as we may, that $u\left(x_{0}\right)=0=\psi\left(x_{0}\right)$. If $r \leq$ $\frac{1}{8} \operatorname{dist}\left(x_{0}, \partial \Omega\right)$ and

$$
\gamma_{0}=\operatorname{osc}\left(\psi, B\left(x_{0}, 8 r\right)\right)
$$

then $\left(u-\gamma_{0}\right)^{+}$is a subsolution and $u+\gamma_{0}$ a nonnegative supersolution in $B\left(x_{0}, 8 r\right)$. Hence we deduce from the weak Harnack inequalities [HKM] 3.34 and 3.59] that

$$
\begin{aligned}
\sup _{B\left(x_{0}, r\right)}\left(u-\gamma_{0}\right) & \leq c\left(f_{B\left(x_{0}, 2 r\right)}\left|\left(u-\gamma_{0}\right)^{+}\right|^{p-1} d x\right)^{1 /(p-1)} \\
& \leq c\left(f_{B\left(x_{0}, 2 r\right)}\left(u+\gamma_{0}\right)^{p-1} d x\right)^{1 /(p-1)} \\
& \leq c \inf _{B\left(x_{0}, 2 r\right)}\left(u+\gamma_{0}\right) \\
& \leq c \gamma_{0} .
\end{aligned}
$$

Keeping in mind that $u \geq \psi \geq-\gamma_{0}$ we conclude

$$
\operatorname{osc}\left(u, B\left(x_{0}, r\right)\right) \leq c \gamma_{0}=c \operatorname{osc}\left(\psi, B\left(x_{0}, 8 r\right)\right) .
$$

Let $r \leq \frac{1}{32} \operatorname{dist}\left(x_{0}, \partial \Omega\right)$ and let $\eta \in C_{0}^{\infty}\left(B\left(x_{0}, 2 r\right)\right)$ be a usual nonnegative cut-off function with $\eta=1$ in $B\left(x_{0}, r\right)$ and $|\nabla \eta| \leq 2 / r$. Then we obtain by applying the Caccioppoli estimate [HKM, 3.29] to $u-\sup _{B\left(x_{0}, 2 r\right)} u$ and (2.2) that

$$
\begin{aligned}
\mu\left(B\left(x_{0}, r\right)\right) & \leq \int_{B\left(x_{0}, 2 r\right)} \eta^{p} d \mu=p \int_{B\left(x_{0}, 2 r\right)} \eta^{p-1} \mathcal{A}(x, \nabla u) \cdot \nabla \eta d x \\
& \leq c\left(\int_{B\left(x_{0}, 2 r\right)}|\nabla u|^{p} \eta^{p} d x\right)^{(p-1) / p}\left(\int_{B\left(x_{0}, 2 r\right)}|\nabla \eta|^{p} d x\right)^{1 / p} \\
& \leq c r^{n-p} \operatorname{osc}\left(u, B\left(x_{0}, 2 r\right)\right)^{p-1} \\
& \leq c r^{n-p} \operatorname{osc}\left(\psi, B\left(x_{0}, 16 r\right)\right)^{p-1} .
\end{aligned}
$$


Now if $x_{0} \in I$ is such that

$$
\operatorname{dist}\left(x_{0}, K\right) \leq r \leq 2 r_{0},
$$

we have the estimate

$$
\mu\left(B\left(x_{0}, r\right)\right) \leq c r^{n-p+\alpha(p-1)},
$$

where $c=c(n, p, M)>0$.

Finally, for $x_{0} \in K$ and $r<r_{0}$, there are two alternatives. Either $B\left(x_{0}, r\right) \cap I=\emptyset$ and thus $\mu\left(B\left(x_{0}, r\right)\right)=0$, or there is $x \in B\left(x_{0}, r\right) \cap I$. In this latter case

$$
\mu\left(B\left(x_{0}, r\right)\right) \leq \mu(B(x, 2 r)) \leq c r^{n-p+\alpha(p-1)}
$$

by (2.3). The lemma is proven.

Remark. Using (1.9) and (2.2), one can easily prove that if $\psi \in C^{0, \alpha}(\Omega)$, then $\hat{R}^{\psi} \in C^{0, \beta}(\Omega)$, where $\beta=\min (\alpha, \kappa)$ and $\kappa>0$ is the constant such that (1.9) holds (see e.g. [HKM, 6.47]).

Proof of Theorem 1.6. Fix a regular set $D \subset \subset \Omega$, for instance a ball. Let $v=\hat{R}^{u}=$ $\hat{R}^{u}(D)$ and

$$
\mu=-\operatorname{div} \mathcal{A}(x, \nabla v) .
$$

Let $K \subset E \cap D$ be compact. Since sets of $n-p$ Hausdorff measure zero $(p \leq n)$ are known to be removable for bounded $\mathcal{A}$-harmonic functions (see e.g. [HKM]), we need only consider the case where $\alpha=(s+p-n) /(p-1)>0$. Since $s=n-p+\alpha(p-1)$ we infer from (1.7) and Lemma 2.1 that

$$
\mu(B(x, r)) \leq c r^{s}
$$

for all $r \leq r_{0}$ and $x \in K$. Because $\mathcal{H}^{s}(K)=0$, we may cover $K$ by balls $B\left(x_{j}, r_{j}\right)$ so that

$$
\mu(K) \leq \sum_{j} \mu\left(B\left(x_{j}, r_{j}\right)\right) \leq c \sum_{j} r_{j}^{s}<\varepsilon,
$$

where $\varepsilon>0$ is given. Consequently, $\mu(E \cap D)=0$ and therefore $\mu=0$, which means that $v$ is $\mathcal{A}$-harmonic in $D[\mathrm{M}, 3.19]$.

Next let $w=\underline{\hat{R}}^{u}(D)$. We similarly find that $w$ is $\mathcal{A}$-harmonic in $D$. Since $v=u=w$ on $\partial D$ by [HKM 9.26], we have that $v=w$ in $D$ by the uniqueness of $\mathcal{A}$-harmonic functions. Since

$$
w \leq u \leq v=w
$$

$u$ is $\mathcal{A}$-harmonic in $D$ and the theorem follows.

\section{Proof of Theorems 1.14 And 1.10}

We recall that $\kappa$ is the constant such that (1.9) holds for every $\mathcal{A}$-harmonic function $h$ in $\Omega$. Then

$$
\int_{B(x, r)}|\nabla h|^{p} d x \leq c\left(\frac{r}{R}\right)^{n-p+p \kappa} \int_{B(x, R)}|\nabla h|^{p} d x,
$$

for each $0<r<R$ with $B(x, R) \subset \Omega$; here $c=c(n, p, \lambda, \Lambda)>0$ (see e.g. $\underline{\mathrm{K}}, 2.1]$ ). 
The following lemma provides the key estimate.

3.2. Lemma. Let $u \in W^{1, p}\left(B\left(x_{0}, R\right)\right)$ be a solution of

$$
-\operatorname{div} \mathcal{A}(x, \nabla u)=\mu,
$$

where $\mu$ is a nonnegative Radon measure such that

$$
\mu\left(B\left(x_{0}, r\right)\right) \leq c_{0} r^{n-p+\alpha(p-1)}
$$

for all $0<r \leq R$. Then for each $0<r<R$ and $\varepsilon>0$ we have

$$
\int_{B\left(x_{0}, r\right)}|\nabla u|^{p} d x \leq c_{1}\left(\left(\frac{r}{R}\right)^{n-p+p \kappa}+\varepsilon\right) \int_{B\left(x_{0}, R\right)}|\nabla u|^{p} d x+c_{2} R^{n-p+p \alpha},
$$

where $c_{1}=c_{1}(n, p, \lambda, \Lambda)>0$ and $c_{2}=c_{2}\left(n, p, \lambda, \Lambda, \alpha, c_{0}, \varepsilon\right)>0$.

Proof. There is no loss of generality in assuming that $r<R / 2$. Let $h$ be the $\mathcal{A}$-harmonic function in $B\left(x_{0}, R\right)$ with $u-h \in W_{0}^{1, p}\left(B\left(x_{0}, R\right)\right)$. Then

$$
\begin{aligned}
& \lambda \int_{B\left(x_{0}, r\right)}|\nabla u|^{p} d x \leq \int_{B\left(x_{0}, r\right)} \mathcal{A}(x, \nabla u) \cdot \nabla u d x \\
& =\int_{B\left(x_{0}, r\right)}(\mathcal{A}(x, \nabla u)-\mathcal{A}(x, \nabla h)) \cdot(\nabla u-\nabla h) d x \\
& \quad+\int_{B\left(x_{0}, r\right)} \mathcal{A}(x, \nabla h) \cdot(\nabla u-\nabla h) d x+\int_{B\left(x_{0}, r\right)} \mathcal{A}(x, \nabla u) \cdot \nabla h d x \\
& \leq \int_{B\left(x_{0}, R\right)}(\mathcal{A}(x, \nabla u)-\mathcal{A}(x, \nabla h)) \cdot(\nabla u-\nabla h) d x \\
& \quad+\Lambda \int_{B\left(x_{0}, r\right)}|\nabla h|^{p-1}|\nabla u|+|\nabla h||\nabla u|^{p-1} d x
\end{aligned}
$$

where we used the structural assumptions (1.3)-(1.5). Since $h$ is $\mathcal{A}$-harmonic with $h-u \in W_{0}^{1, p}\left(B\left(x_{0}, R\right)\right)$ and thus quasiminimizes the $p$-Dirichlet integral, we have by using Adams' inequality (see [AH], Thm 7.2.2] or [Z, Thm 4.7.2]) that

$$
\begin{aligned}
\int_{B\left(x_{0}, R\right)} & (\mathcal{A}(x, \nabla u)-\mathcal{A}(x, \nabla h)) \cdot(\nabla u-\nabla h) d x=\int_{B\left(x_{0}, R\right)}(u-h) d \mu \\
& \leq c R^{(p-1)(n-p+\alpha p) / p}\left(\int_{B\left(x_{0}, R\right)}|\nabla u-\nabla h|^{p} d x\right)^{1 / p} \\
& \leq c R^{n-p+\alpha p}+\frac{\lambda}{2} \varepsilon \int_{B\left(x_{0}, R\right)}|\nabla u|^{p} d x,
\end{aligned}
$$

where we also used Young's inequality. The remaining integrals on the right of (3.3) do not exceed

$$
\begin{aligned}
& \frac{\lambda}{2} \int_{B\left(x_{0}, r\right)}|\nabla u|^{p} d x+c \int_{B\left(x_{0}, r\right)}|\nabla h|^{p} d x \\
& \leq \frac{\lambda}{2} \int_{B\left(x_{0}, r\right)}|\nabla u|^{p} d x+c\left(\frac{r}{R}\right)^{n-p+p \kappa} \int_{B\left(x_{0}, R\right)}|\nabla h|^{p} d x \\
& \leq \frac{\lambda}{2} \int_{B\left(x_{0}, r\right)}|\nabla u|^{p} d x+c\left(\frac{r}{R}\right)^{n-p+p \kappa} \int_{B\left(x_{0}, R\right)}|\nabla u|^{p} d x,
\end{aligned}
$$


where we also employed (3.1) and the quasiminimizing property of $\mathcal{A}$-harmonic functions. Plugging these estimates in (3.3) we arrive at

$$
\begin{aligned}
\int_{B\left(x_{0}, r\right)}|\nabla u|^{p} d x \leq c R^{n-p+\alpha p}+\varepsilon \int_{B\left(x_{0}, R\right)}|\nabla u|^{p} d x \\
+c\left(\frac{r}{R}\right)^{n-p+p \kappa} \int_{B\left(x_{0}, R\right)}|\nabla u|^{p} d x .
\end{aligned}
$$

The lemma follows.

Proof of Theorem 1.14. If $B\left(x_{0}, 4 R\right) \subset \Omega$, then by appealing to [G, Lemma III.2.1, p. 86] Lemma 3.2 yields

$$
\int_{B\left(x_{0}, r\right)}|\nabla u|^{p} d x \leq c\left(\frac{r}{R}\right)^{n-p+p \alpha}
$$

for $r<R$. Thus $u \in C^{0, \alpha}(\Omega)$ by the Dirichlet growth theorem $\mathrm{G}$, Theorem III.1.1, p. 64].

Proof of Theorem 1.10. Let $\kappa$ be the number as in Theorem 1.14. Let $K \subset E$ be compact with $\mathcal{H}^{n-p+\alpha(p-1)}(K)>0$. Frostman's lemma ([AH, 5.1.12], [C]) gives us a nonnegative Radon measure $\mu$ living on $K$ with $\mu(K)>0$ and $\mu(B(x, r)) \leq$ $r^{n-p+\alpha(p-1)}$. Any solution $u \in W_{\text {loc }}^{1, p}(\Omega)$ to

$$
-\operatorname{div} \mathcal{A}(x, \nabla u)=\mu
$$

is $\mathcal{A}$-harmonic in $\Omega \backslash E[\mathrm{M}, 3.19]$ and $u \in C^{0, \alpha}(\Omega)$ by Theorem 1.14, but $u$ fails to have an $\mathcal{A}$-harmonic extension to $\Omega$, since $\mu(K)>0$.

\section{REFERENCES}

[AH] Adams, D. R., and Hedberg, L.I., Function spaces and potential theory, Springer, 1996. $\operatorname{MR}$ 97j:46024

[BK] Buckley, S. M. and Koskela, P., On the fusion problem for degenerate elliptic equations II, Comment. Math. Univ. Carolin. 40 (1999), 1-6. MR 2000j:35078

[C] Carleson, L., Selected problems on exceptional sets, Van Nostrand, 1967. MR 37:1576

[DM] David, G. and Mattila, P., Removable sets for Lipschitz harmonic functions in the plane, Revista Mat. Iberoamericana 16 (2000), 137-215. CMP 2000:15

[G] Giaquinta, M., Multiple integrals in the calculus of variations and nonlinear elliptic systems, Princeton University Press, 1983. MR 86b:49003

[HK] Heinonen, J. and Kilpeläinen, T., A-superharmonic functions and supersolutions of degenerate elliptic equations, Ark. Mat. 26 (1988), 87-105. MR 89k:35079

[HKM] Heinonen, J., Kilpeläinen, T., and Martio, O., Nonlinear potential theory of degenerate elliptic equations, Oxford University Press, Oxford, 1993. MR 94e:31003

[K] Kilpeläinen, T., Hölder continuity of solutions to quasilinear elliptic equations involving measures, Potential Analysis 3 (1994), 265-272. MR 95j:35071

[KM] Kilpeläinen, T. and Malý, J., The Wiener test and potential estimates for quasilinear elliptic equations, Acta Math. 172 (1994), 137-161. MR 95a:35050

[KKM] Kilpeläinen, T., Koskela, P., and Martio, O., On the fusion problem for degenerate elliptic equations, Comm. PDE 20 (1995), 485-497. MR 96e:35055

[KM1] Koskela, P. and Martio, O., Removability theorems for quasiregular mappings, Ann. Acad. Sci. Fenn. Ser. A I Math. 15 (1990), 381-399. MR 92i:30020]

[KM2] Koskela, P. and Martio, O., Removability theorems for solutions of degenerate elliptic partial differential equations, Ark. Mat. 31 (1993), 339-353. MR 95c:35082

[L] Lieberman, G.M., Regularity of solutions to some degenerate double obstacle problems, Indiana Univ. Math. J. 40 (1991), 1009-1028. MR 92m:35102 
[M] Mikkonen, P., On the Wolff potential and quasilinear elliptic equations involving measures, Ann. Acad. Sci. Fenn. Ser. A I. Math. Dissertationes 104 (1996), 1-71. MR 97e:35069

[TW] Trudinger, N. and Wang, X.J., On the weak continuity of elliptic operators and applications to potential theory, Preprint (2000), (http://www.maths.anu.edu.au/research.reports/mrr/00.018/MRR00-018.dvi.gz).

[Z] Ziemer, W. P., Weakly differentiable functions, Springer-Verlag, 1989. MR 91e:46046

Department of Mathematics, University of Jyväskylä, P.O. Box 35, 40351 Jyväskylä, FINLAND

E-mail address: terok@math.jyu.fi

Department of Mathematics, University of Jyväskylä, P.O. Box 35, 40351 Jyväskylä, FinLAND

E-mail address: zhong@math.jyu.fi 